\title{
Research on 5G Millimeter Wave Hybrid Precoding Algorithm
}

\author{
Xu Shuang \\ School of communication Engineering \\ Chengdu University of Information Technology \\ ChengDu, China
}

\begin{abstract}
This paper mainly studies the precoding technology through the combination of two key technologies of 5G, and proposes a new precoding method to compare with the previous precoding technology. The results show that the new precoding method is better. Keywords: 5G;MIMO; Millimeter; styling; Precoding
\end{abstract}

\section{INTRODUCTION}

Now, the spectrum of the low frequency band has been basically allocated, and it is occupied by various important communication services, and it is impossible to repeatedly allocate it to 5G. Even if these bands can be allocated 5G, their spectrum resources cannot meet the 5G bandwidth requirements. In order to adapt to the development of the future network, people have turned their attention to the millimeter wave resources in the high frequency band.

Communication on millimeter wave frequencies has the following features.First of all, there are a large number of unlicensed spectrum resources in the millimeter wave band, and the frequency band width reaches several hundred GHz. Since there are a large number of frequency bands available for distribution, communication over millimeter wave frequencies has an extremely broad prospect. High-speed wireless communication using the spectrum resources of the millimeter-wave band can significantly increase the system capacity of the communication system and effectively solve the current spectrum resource shortage problem.

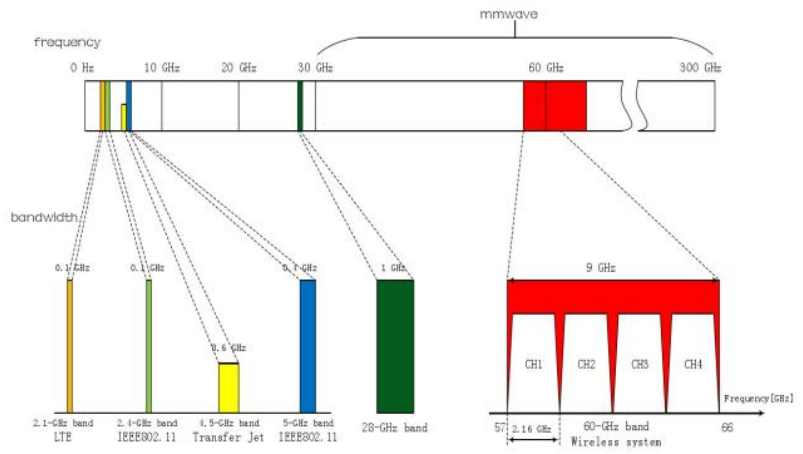

Figue.1 0-300GHz Spectrum resource allocation map

Second, the millimeter wave has a very short wavelength and a wavelength of only 1 to $10 \mathrm{~mm}$. An array of many antenna elements can be integrated into a small area, helping to reduce the size of the antenna device and reduce application costs.With multi-antenna technology, millimeter waves can achieve good beam directivity, which can reduce electromagnetic interference between users, reduce system energy consumption, and achieve environmentally friendly communication. It is not difficult to conclude that the application of millimeter wave technology is inseparable from multi-antenna technology, and the two are complementary.
Multi-antenna technology, or MIMO technology, is one of the key technologies of 4G. The MIMO technology utilizes the diversity formed by the multi-antenna deployment at the transceiver end to improve the reliability of the system, and can also utilize multiple channels formed by multi-antenna deployment to improve the transmission rate and spectrum efficiency of the system. In 5G, small-scale MIMO is not suitable for high-speed and large-capacity communication. Researching systems using large-scale antennas is an important direction in the current 5G.

In conventional low-band MIMO systems, precoding to eliminate interference between different data streams is implemented in the digital domain. In digital precoding, each antenna is assigned a corresponding RF chain (including devices such as digital-to-analog converters). After rising to the millimeter-wave band, it is necessary to use devices with a wide range of system bandwidth. Expensive, and the power consumption of a single link is much higher than at low frequencies. If a separate RF link is configured for each antenna in a millimeter wave system, the overall cost and energy consumption of the RF link will not be negligible. For example, in a 64-antenna millimeter wave MIMO system using a conventional digital precoding scheme, the power requirement of the RF link is $16 \mathrm{~W}$. To solve this problem, the researchers explored the structure of analog and digital hybrid precoding systems.

\section{MILLIMETER WAVE HYBRID PRECODING TECHNOLOGY}

In hybrid precoding technology, the precoding process of the system is decomposed into the analog domain and the digital domain. The RF link is configured only in the digital domain, and the phase shifter or switch matrix is configured in the analog domain, thereby greatly reducing the number of RF links. . Research shows that hybrid precoding does not cause much loss to system performance, but it can significantly improve the system's power consumption, thus reducing the information transmission cost of the communication system. 


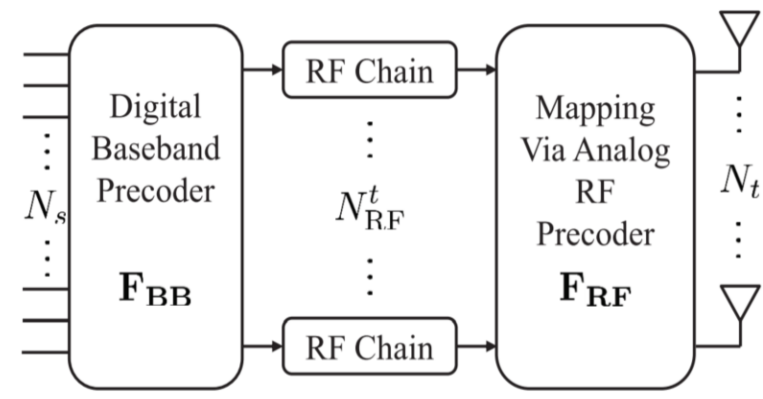

Figue.2 Millimeter wave hybrid precoding architecture

Study a hybrid architecture millimeter-wave MIMO system as depicted in Figure 2. The transmitter has a root antenna and Radio link. A radio link with $N_{T}$ at the receiving end. Root antenna and $N_{R F}$. Radio link. Between the transmitting end and the receiving end.The $N_{s}$ and strip data streams are transmitted and have the following relationship $N_{R F} \leq N_{T}$, $N_{R F} \leq N_{R}$. Configured at the transmitting end There is an analog precoding matrix $F_{R F}$, digital precoding matrix $F_{B B}$, and an analog combining matrix $W_{R F}$ is configured at the receiving end,Digital merge matrix $W_{B B}$.

Data is transmitted using one of the millimeter wave MIMO systems described above. Let $\mathrm{S}$ be the transmitted symbol vector, then the signal $\mathrm{X}$ on the transmitting antenna can be expressed as $X=F_{R F} F_{B B} \mathrm{~s}$. The transmitted signal $\mathrm{x}$ is transmitted to the receiving end through the transmission of the millimeter wave MIMO channel, and is processed by the combining matrix to obtain the signal Y. This transmission process is described by follow equation .

$$
\mathrm{y}=\sqrt{\rho} W_{B B}^{H} W_{R F}^{H} H F_{R F} F_{B B} \mathrm{~s}+W_{B B}^{H} W_{R F}^{H} \mathbf{n}
$$

$\rho$ is the power of the received signal. $\mathrm{n}$ is a Gaussian with a mean of 0 and a covariance matrix of $\sigma_{\mathrm{n}}^{2} I_{N_{R}}$ noise vector. The work that hybrid precoding needs to do is to design the optimal performance precoding matrix $F_{R F}, F_{B B}$ and merge matrix $W_{R F}, W_{B B}$ under the condition of meeting the relevant hardware constraints.

\section{PROPOSE ALGORITHM}

Algorithm1 Proposed algorithm with RSD.

\section{Require: $F_{\text {opt }}$}

1: Initialize $\mathrm{x}^{0}$

2 : for $\mathrm{k}=0,1,2, \ldots$ do

3: Inverse-vectorize $\mathrm{x}^{\mathrm{k}}$ as $F_{R F}^{\mathrm{k}}=\operatorname{vec}^{-1}\left(\mathrm{x}^{\mathrm{k}}\right)$.

4: Compute $F_{B B}^{F}=\left(F_{R F}^{K}\right) F_{\text {opt }}$.

5: Compute the Euclidean gradient $\nabla \mathbf{f}\left(\mathrm{x}^{\mathrm{k}}\right)$ using (5).

6: Compute the Riemannian gradient $\xi^{\mathrm{k}}=P_{\mathrm{x}^{\mathrm{k}}} \nabla \mathrm{f}\left(\mathrm{x}^{\mathrm{k}}\right)$.

7: Find the step size $\alpha^{\mathrm{k}}$, and updat $\mathrm{x}_{\mathrm{x}^{\mathrm{k}+1}}=R_{\mathrm{x}^{\mathrm{k}}}\left(-\alpha^{\mathrm{k}} \xi^{\mathrm{k}}\right)$.

$$
\begin{aligned}
& \text { 8: end for } \\
& \text { 9: Output } \\
& \qquad F_{R F}=\operatorname{vec}^{-1}\left(\mathrm{x}^{K+1}\right), \quad F_{B B}=\frac{\sqrt{N_{B}}}{\left\|F_{R F} F_{B B}^{K}\right\|_{F}} \hat{F}_{B B}^{K}
\end{aligned}
$$

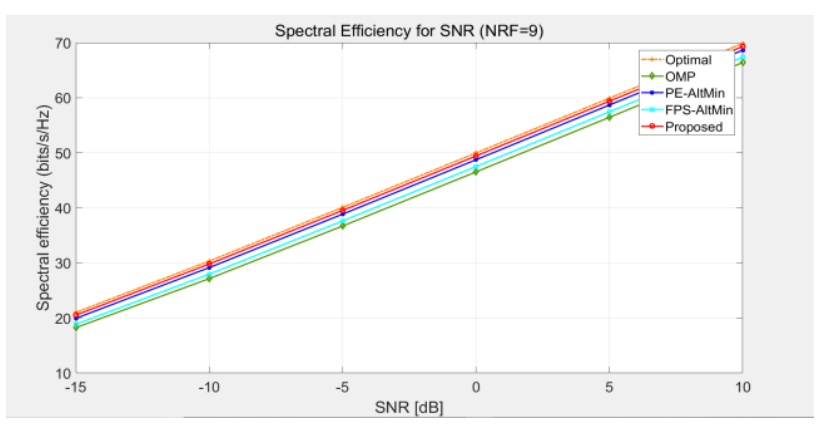

Figue. $3 N_{S}=6, N_{R F}=9, N_{\mathrm{t}}=144, N_{\mathrm{r}}=36$

Since the following procedure is analogous to that of the narrowband mmWave system,it is omitted. Lastly,the overall algorithm is summarized in Algorithm 1, where, for simplicity, the RSD is used. It is noteworthy that, comparing with AltMin, the updates of and in the proposed algorithm are mutually integrated into one single-loop structure, achieving faster convergence of the proposed algorithm

\section{4. .CONCLUSIONS}

Millimeter-wave massive MIMO is an important direction for future development. It can be further explored in precoding to provide more protection for the future $5 \mathrm{G}$..

\section{REFERENCES}

[1] Bowman, M., Debray, S. K., and Peterson, L. L. 1993. X.

Yu, J.-C. Shen, J. Zhang, and K. B. Letaief, “Alternating minimization algorithms for hybrid precoding in millimeter wave MIMO systems," IEEE Journal on Selected Areas in

Communications, vol. 10, no. 3, pp. 485-500, 2016

[2]R.W.Daniels,R.C.Heath, "60GHzwirelesscommunications: Emerging requirements and design recommendations," IEEE Veh. Technol. Mag., vol. 2, no. 3, pp. 41-50, 2007

[3]P.-A. Absil and K. A. Gallivan, "Joint diagonalization on the oblique manifold for independent component analysis," in ICASSP, 2006

[4]O. E. Ayach, S. Rajagopal, S. Abu-Surra, Z. Pi, and R. W. Heath, "Spatially sparse precoding in millimeter waveMIMOsystems," IEEETrans.WirelessCommun., vol. 13, no. 3, pp. 1499-1513, 2014.

[5]Hiroyuki Kasai,"Fast Optimizati on Algorithm complex 
International Journal of Computer Applications Technology and Research Volume 8-Issue 07, 276-278, 2019, ISSN:-2319-8656 